\title{
Fingerprint Recognition Based on Combined Features
}

\author{
Yangyang Zhang, Xin Yang, Qi Su, and Jie Tian \\ Center for Biometrics and Security Research, Key Laboratory of Complex Systems \\ and Intelligence Science, Institute of Automation, Chinese Academy of Sciences, \\ Graduate School of the Chinese Academy of Sciences, \\ P.O.Box 2728 Beijing 100080 China \\ tiandieee.org, jie.tian@ia.ac.cn \\ http://www.fingerpass.net
}

\begin{abstract}
In this paper, we represent the fingerprint with a novel local feature descriptor, which is composed of minutia, the sample points on associated ridge and the adjacent orientation distribution. Then a novel fingerprint recognition method is proposed combining the orientation field and the local feature descriptor. We compare two descriptor lists from the input and template fingerprints to calculate a set of transformation vectors for fingerprint alignment. The similarity score is evaluated by fusing the orientation field and the local feature descriptor. The experiments have been conducted on three large-scale databases. The comparison results approve that our algorithm is more accurate and robust than previous methods based on the minutiae or ridge features, especially for those poor-quality and partial fingerprints.
\end{abstract}

Keywords: orientation field, local feature descriptor, fingerprint alignment, fusing, similarity score.

\section{Introduction}

Fingerprints are ridge patterns flowing on the surface of fingers, which have been increasingly used for individual identification in the civilian's daily life due to the uniqueness, permanence and universality. Previously researchers have proposed many kinds of fingerprint recognition algorithms. The most popular methods are based on point features (called minutiae), which extract minutiae sets from the input fingerprint and compare them with those from the template fingerprint to calculate the similarity score [1] [2]. The minutiae-based features are simple for storage and effective for comparison, but there are still some limits in fingerprint recognition. First, the accuracy of minutiae extraction relies on local quality of fingerprint. Second, minutiae cannot characterize the overall pattern of a fingerprint, and it is hard to further improve the performance. So researchers proposed other representations of fingerprints to resolve these problems, such as texture, ridge structure, orientation field and so on.

The ridge feature observes more information in the whole region of fingerprint and reinforces the individuality of fingerprint considerably. Tian et. al [3] constructed the minutia-simplex which contains a pair of minutiae with the associated local ridge information to estimate the parameters of affine transform and calculate the similarity 
score. Feng et. al [10] established both the ridge correspondences and the minutia correspondences between two fingerprints. The method realized fingerprint verification after alignment by incrementally matching ridges and minutiae. Both of the algorithms survive the problem of the alignment inaccuracy, which influences their performance in some extent. Another kind of important features is orientation field in fingerprint processing. It describes the global structure of the fingerprint ridge pattern. Gu et. al [5] represented and verified the fingerprints combing both minutiae and model-based orientation field. The algorithm performs well on FVC2002 databases, but in reality it's difficult to estimate the global model for some partial fingerprints.

In this paper we represent the fingerprint with a novel local feature descriptor, which is composed of minutia, the sample points on associated ridge and the adjacent orientation distribution. A novel approach is introduced for fingerprint recognition by fusing the orientation field and the local feature descriptor. First, we align two fingerprints by the local feature descriptor and compute the transformation parameters including the translation and rotation. Second, we combine the local feature descriptor and orientation field feature in fingerprint matching. The comparison of orientation field can reduce the false accepted rate to accelerate the matching process. The similarity score is calculated based on combined features with the SUM rule, which is the most accurate in the experiments. We conduct a set of experiments on three large-scale databases and make comparisons with the state-of-the-arts. The results show that the fusion of local feature descriptor and orientation field can certainly obtain better fingerprint authentication performance than previous methods based on minutiae or ridge features.

The rest of the paper is organized as follows: Section 2 proposes the fingerprint representation with the local feature descriptor. Section 3 presents the method of alignment by using the local feature descriptor. Section 4 describes the process of fingerprint matching based on both the orientation field and the local feature descriptors. The experimental results for our algorithm are displayed in section 5 and Section 6 summarizes our researches.

\section{Fingerprint Representation}

We represent the fingerprint with a set of local feature descriptors, each of which consists of a minutia, the sample points on its associated ridge and the adjacent orientation distribution. The minutiae set denoted by $\left\{m^{k}=\left(x_{m}^{k}, y_{m}^{k}, \theta_{m}^{k}\right) \mid 1 \leq k \leq K\right\}$ are extracted from the thinned fingerprint image with the conventional methods, where $m^{k}$ is the $k$ th minutia, $K$ is the number of minutiae, $\left(x_{m}^{k}, y_{m}^{k}\right)$ and $\theta_{m}^{k} \in[0, \pi)$ are respectively the location and orientation for $m^{k}$. In this section, we mainly discuss how to construct the local feature descriptor for the minutiae of different types.

According to the traditional methods, minutiae are classified into two types: ridge termination and bifurcation. In practical it is rather difficult to distinguish them because of the noise and environment during fingerprint capture. The ridge ending is possibly mistaken as the bifurcation due to ridge conglutination, while the bifurcation may turn into the ending due to the broken ridge.

To solve this problem, we sample both the associated ridge and valley in a constant interval to express the ridge features in a common form. The ridge feature structure is 
constructed with the information of sample points assigned to minutia as shown in Fig.1 We define the set of ridge structures $R=\left\{r^{k}\right\}_{k-1}^{K}$ in a fingerprint as follows:

$$
\begin{gathered}
r^{k}=\left\{\left\{x_{t, i}^{k}, y_{t, i}^{k}, \text { len }_{t, i}^{k}, \Delta \phi_{t, i}^{k}\right\}_{t=0,1}\right\}_{i=1}^{L_{t}^{k}} . \\
\Delta \phi_{t, i}^{k}=d\left(\theta_{t, i}^{k}, \theta_{m}^{k}\right) \\
d\left(\theta_{1}, \theta_{2}\right)= \begin{cases}\theta_{1}-\theta_{2} & \text { if } a b s\left(\theta_{1}-\theta_{2}\right) \leq \pi / 2 \\
\theta_{1}-\theta_{2}-\pi & \text { if }\left(\theta_{1}-\theta_{2}\right)>\pi / 2 \\
\theta_{1}-\theta_{2}+\pi & \text { if }\left(\theta_{1}-\theta_{2}\right)<-\pi / 2\end{cases}
\end{gathered}
$$

where $r^{k}$ is the local ridge structure belonging to the $k t h$ minutia. $t \in\{0,1\}$ denotes the sampling type. When the sample points on the ridge for bifurcation or on the valley for ending, the value of $t$ is set 0 , otherwise, it's set $1 . L_{t}^{k}$ denotes the number of sample points. $\left(x_{t, i}^{k}, y_{t, i}^{k}\right)$ are the coordinates of the sample points. $l e n_{t, i}^{k}, \theta_{t, i}^{k}$ are the distance and orientation from the $k t h$ minutia $m^{k}$ to the $i t h$ sample point and $\theta_{t, i}^{k} \in[0, \pi)$. $d\left(\theta_{1}, \theta_{2}\right)$ is called the orientation distance taking the value in $[-\pi / 2, \pi / 2]$. The ridge and valley is traced until encountering another minutia or arriving the border of fingerprint. If the sampling number $L_{t}^{k}$ is less than $l T h r$ (In our experiments, $l T h r=3$ ), the associated ridge structure is considered false and discarded.

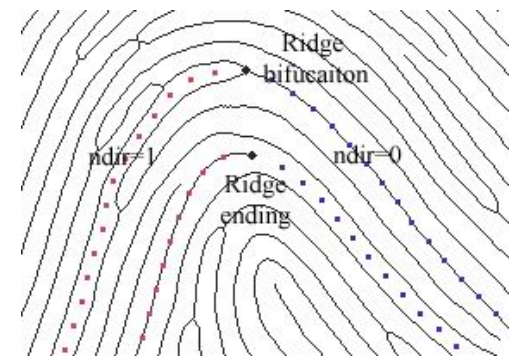

(a)

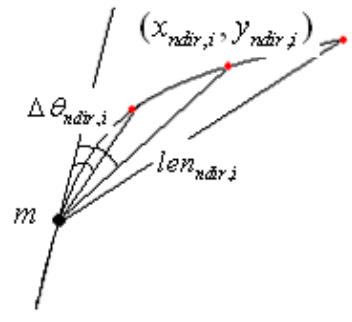

(b)

Fig. 1. (a) sampling ridges in two directions for two types of minutiae, (b) ridge structure in one direction for the minutia $\mathrm{m}$

We also characterize each minutia with a structure that comprises information for the orientation field in a broad region around the minutia point. The structure comprises the sampling points equally distributed in a matrix pattern centered at each minutia as illustrated in Fig. 2. According to the sampling principle, the sampling interval $\mathrm{v}$ is selected as $2 \tau$ ( $\tau$ is the average fingerprint ridge width) to obtain the best tradeoff between decreasing storage and preserving information.

We define the set of orientation structures $V=\left\{v_{k}\right\}_{k=1}^{K}$ in the fingerprint as follows:

$$
v_{k}=\left\{\left(\Delta \varphi_{i, j}^{k}\right)_{j=-N_{k}}^{N_{k}}\right\}_{i=-N_{k}}^{N_{k}}
$$




$$
\Delta \varphi_{i, j}^{k}=d\left(\theta_{i, j}^{k}, \theta_{m}^{k}\right)
$$

where $K$ is the number of minutiae, $2 N_{k}+1(1 \leq k \leq K)$ is the dimension of sampling matrix around the $k t h$ minutia and each sampling matrix consist of $\left(2 N_{k}+1\right) *\left(2 N_{k}+1\right)$ points $p_{i, j}^{k}\left(-N_{k} \leq i, j \leq N_{k}\right) . \theta_{i, j}^{k} \in[0, \pi)$ denote the orientation at each sample point.

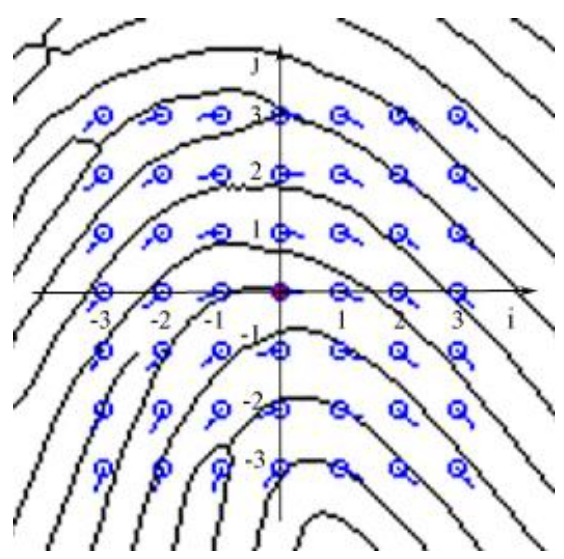

Fig. 2. The orientation distribution around a minutia (the red point), the circle denote the sample points $P_{i, j}\left(N_{k}=3, \mathrm{v}=16\right)$

The local feature descriptor is proposed for each minutia including its location and orientation, the associated ridge structure and the adjacent orientation structure. We represent the fingerprint with a set of local feature descriptors as follows:

$$
M=\left\{M^{k} \mid 1 \leq k \leq K\right\} \text { and } M^{k}=\left\{m^{k}, r^{k}, v^{k}\right\}
$$

\section{Fingerprint Alignment}

The alignment stage determines the correct transformation between two fingerprints, which plays a crucial role in fingerprint recognition. In our approach, we realize the alignment by finding $N$ most similar local feature descriptor pairs and calculating the corresponding transformation vectors as candidates. Compared with using the best one for alignment, the muti-candidates method is more robust to those incorrect and displaced descriptors.

A local similarity function between two descriptors $M^{f}=\left\{m^{f}, r^{f}, v^{f}\right\}$ and $M^{t}=$ $\left\{m^{f}, r^{f}, v^{f}\right\}$ is defined as:

$$
\operatorname{Sm}\left(M^{f}, M^{t}\right)=\operatorname{Sr}\left(r^{f}, r^{t}\right) * S v\left(v^{f}, v^{t}\right)
$$

where $S r\left(r^{f}, r^{t}\right)$ and $S v\left(v^{f}, v^{t}\right)$ denote the similarity of ridge and orientation structure.

In the local descriptor, $\operatorname{len}_{t, i}^{k}, \Delta \phi_{t, i}^{k}$ and $\Delta \varphi_{t, i}^{k}$ describe the transformation-invariant relative features of $M^{k}$, while $\left(x_{m}^{k}, y_{m}^{k}, \theta_{m}^{k}\right), \theta_{t, i}^{k}$ and $\theta_{i, j}^{k}$ represent the transformationvariant relative features. We utilize the transform-invariant parts of the local feature 
descriptor for their similarity calculation. The similarity functions of ridge and orientation structure are given in an exponential form [7]:

$$
\begin{gathered}
S r\left(r^{f}, r^{t}\right)=\frac{1}{N_{p}} \sum_{p \in P} \exp \left\{-\frac{1}{\mu}\left(\alpha *\left|\operatorname{len}_{p}^{f}-\operatorname{len}_{p}^{t}\right|+(1-\alpha) *\left|\Delta \phi_{p}^{f}-\Delta \phi_{p}^{t}\right|\right)\right\} \\
S v\left(v^{f}, v^{t}\right)=\frac{1}{N_{q}} \sum_{q \in Q} \exp \left\{-\frac{1}{\omega} *\left|\Delta \varphi_{q}^{f}-\Delta \varphi_{q}^{t}\right|\right\}
\end{gathered}
$$

where $P=\{(t, i)\}$ denote the overlapped part of the sample points on the ridges. $Q=\{(i, j)\}$ indicate the overlapped part of the sample points of orientation field. $N_{p}$ and $N_{q}$ are the size of $P$ and $Q . \alpha \in[0,1]$ adjusts the scale difference between the distance and orientation. The values of $\mu, \omega$ are empirically set in training.

Let $F=\left\{M^{f i}\right\}_{f i=1}^{K^{f}}$ and $T=\left\{M^{t i}\right\}_{t i=1}^{K^{t}}$ denote the descriptor sets detected from the input and template fingerprints. The similarity score is calculated between any pair of local feature descriptors $M^{f i}$ and $M^{t i}$. A relative array $C$ is established to store these index pairs $(f i, t i)$ that satisfy $S m\left(M^{f t}, M^{t i}\right)>S m T h r(S m T h r$ is an experimental variant) in the decrease order of their similarity values.

We construct the alignment candidate set $L$ to store the transformation vectors $L^{n}$ between these descriptor pairs, which have the largest $N$ similarity score $L=\left\{l_{n}\right\}_{n=1}^{N}$. For any pair of $M^{f i}$ and $M^{t i}$ in the candidate set, we average the difference of the position and orientation between two minutiae and the corresponding sample point pairs on the ridges to calculate their transformation vector which includes the translation and rotation parameters, $l^{n}=(d x, d y, d \theta)^{T}$ :

$$
\begin{gathered}
d \theta=\frac{1}{N_{p}} \sum_{p \in P} \theta_{p}^{t}-\theta_{p}^{f} \\
{\left[\begin{array}{l}
d x \\
d y
\end{array}\right]=\frac{1}{N_{p}} \sum_{p \in P}\left(\left[\begin{array}{l}
x_{p}^{t} \\
y_{p}^{t}
\end{array}\right]-R_{d \theta} *\left[\begin{array}{l}
x_{p}^{f} \\
y_{p}^{f}
\end{array}\right]\right)}
\end{gathered}
$$

Where $R_{d \theta}$ is a $2 * 2$ operator of clockwise rotating with $d \theta . P$ and $N_{p}$ are defined in formula 8

\section{Fingerprint Matching}

\subsection{Matching Based on Orientation Field}

In our approach the block orientation field is utilized to avoid misaligning two fingerprints, it measures the similarity of their global ridge patterns. For each transformation vector in the candidate set, we align the orientation fields extracted from the enhanced fingerprints, where the backgrounds have been excluded. The overlapped area A is partitioned into a lattice of blocks of size $n * n$ ( $n$ is decided by ridge frequency and fingerprint quality). 
For each block $B$, let $g_{s}=\left(g_{s}^{x}, g_{s}^{y}\right)$ denotes the gradient intensity at each site $s$. We evaluate the local quality of the fingerprint by using the covariance matrix $J$ and the normalized coherence measure $\widetilde{k} \in[0,1]$ in the block $B$ as follows [8]:

$$
\begin{gathered}
J=\frac{1}{n^{2}} \sum_{s \in B} g_{s} g_{s}^{T}=\left[\begin{array}{ll}
j_{11} & j_{12} \\
j_{21} & j_{22}
\end{array}\right] \\
\widetilde{k}=\frac{j_{11}^{2}-j_{22}^{2}+4 j_{12}^{2}}{\left(j_{11}+j_{22}\right)^{2}}
\end{gathered}
$$

The similarity score of two orientation fields can be computed as the weighted average of the block-wise similarity measures:

$$
\begin{gathered}
S o\left(O^{f}, O^{t}\right)=\frac{1}{\sum_{B \in A} \widetilde{k}_{B}^{f} * \widetilde{k}_{B}^{t}} \sum_{B \in A} S\left(B^{f}, B^{t}\right) * \widetilde{k}_{B}^{f} * \widetilde{k}_{B}^{t} \\
S\left(B^{f}, B^{t}\right)=\exp \left(-\frac{1}{v} *\left|d\left(\bar{\theta}_{B}^{f}, \bar{\theta}_{B}^{t}\right)\right|\right)
\end{gathered}
$$

where $\bar{\theta}_{B}^{f}, \bar{\theta}_{B}^{t}$ are the average orientation in the block $B$ of two fingerprints, $d\left(\bar{\theta}_{B}^{f}, \bar{\theta}_{B}^{t}\right)$ denotes the orientation distance as defined in the formula $3 v$ is experimentally selected based on the training results.

If $S o\left(O^{f}, O^{t}\right)<S o T h r$, the corresponding local descriptor pair is considered misaligned and discarded from the candidate set, which reduces the false accepted rate and accelerates the matching process. SoThr is selected according to false reject rate (in our method at $F R R=0.1 \%$ ) in training.

\subsection{Matching Based on Local Feature Descriptors}

After refining the alignment candidates with the block orientation field, we apply each residual transformation vectors $V^{n}$ in the candidate set $V$ to the two descriptor sets $F$ and $T$ from the input and template fingerprints. The similarity score of fingerprints is evaluated based on local descriptor as the following stages:

- Initialize the set $D P$ to store the corresponding matched descriptor pairs.

- Convert the descriptor set $T$ in the template fingerprint into the aligned descriptor set $T^{\prime}$ with the transformation vector.

- Represent all the minutiae from the two sets $F$ and $T^{\prime}$ in a polar coordinate system whose origin is set at the minutia in $M^{f i}$. Because of the non-linear deformation and the error in feature extracting, the corresponding minutiae from two fingerprints can't be exactly overlapped. If two minutiae fall in the same changeable tolerance box [8], which means, their Euclidean distance and orientation difference don't exceed the value $\delta d$ and $\delta \theta$, two corresponding descriptors are considered as the matched pair. We delete those descriptor pairs that don't locate in the same tolerance box from the relative array $C$ mentioned in section 3 .

- Construct the set of matched descriptor pairs $D P$ with the dynamic programming method. The descriptor pair $\left(M^{f m}, M^{t m}\right)$ that has the largest similarity value is inserted to the set $D P$. All the pairs comprising the two descriptors are discarded from the array $C$. This step is repeated until the array is empty. 
- Evaluate the global similarity score between all the matched descriptor pairs. Considering only the descriptors in the overlapped area after alignment, the similarity is computed as follows:

$$
S p(F, T)=\frac{1}{M} * \sum_{i=1}^{M} S m\left(M^{f m[i]}, S m\left(M^{t m[i]}\right) * \frac{\left(\sum_{i=1}^{M} N s[i]\right)^{2}}{N s^{f} * N s^{t}}\right.
$$

where $M$ is the number of the matched descriptor pairs $(f m[i], t m[i]) . N s[i]$ is the number of the matched sample points in the ith descriptor pair. $N s^{f}$ and $N s^{t}$ respectively denote the number of all the sample points from the two descriptor sets $F$ and $T^{\prime}$ in the overlapped area after fingerprint alignment.

\subsection{Evaluating Combined Similarity Score}

Fusing different features in matching stage can improve the fingerprint authentication performance. The detailed analysis on classifier fusion can be seen in the literatures [9] [10]. We combine the orientation field and local feature descriptors by different strategies in score level. The combined similarity score is evaluated in our approach using four different decision-level fusion rules as follows:

Max rule: Score $=\max (S p, S o)$

Min rule: $S$ core $=\min (S p, S o)$

Product rule: Score $=S p * S o$

Sum rule: Score $=q * S p+(1-q) * S o$, where $q \in[0,1]$ adjusts the weight fractions between the similarity of orientation fields and local descriptors, it is selected based on the quality of fingerprints.

Table 1. Comparison of the performance on FVC2004 DB1

\begin{tabular}{c|c|c|c|c|c|c}
\hline FVC2004 DB1 & Only orientation-based & Only descriptors-based & Max & Min & Product & Sum \\
\hline EER $(\%)$ & 6.53 & 4.74 & 4.56 & 4.95 & 4.35 & 3.49 \\
\hline FMR100 $(\%)$ & 17.33 & 11.97 & 10.42 & 11.72 & 9.71 & 6.21 \\
\hline FMR1000(\%) & 27.61 & 23.50 & 23.29 & 19.47 & 15.36 & 19.04 \\
\hline ZeroFMR(\%) & 38.43 & 28.97 & 28.97 & 23.45 & 19.93 & 24.61 \\
\hline
\end{tabular}

We evaluate the set of combined similarity score according to each transformation vector in candidates. The maximum value is selected as the final matching score. Experimental results performed on DB1 of FVC2004 with four fusion rules are displayed in Table 1 It is obvious that the performance of the Sum rule is the best, while the performance of the Min rule is the worst. Hence, the Sum rule is selected in our algorithm to evaluate the combined similarity score between the template and input fingerprints.

\section{Experimental Results}

The experiments have been conducted on FVC2004 [11] and one database of our lab. Each database of FVC2004 consists of 800 fingerprint images (100 fingers 8 
impressions per finger) captured at a resolution of about 500dpi. Databases emphasize on distortion, dry and wet fingerprints especially in DB1 and DB3 of FVC2004, which are more difficult than FVC2000/FVC2002. Therefore our algorithm is evaluated on DB1 and DB3 of FVC2004. We also establish the database to test the efficiency of our algorithm for poor-quality and partial fingerprints. The database comprises 1680 fingerprints (140 fingers 12 impressions per finger) acquired with an electric field sensor "Authentec 4000". The image size is $96 * 96$ pixels at a resolution of $250 \mathrm{dpi}$. Due to the low resolution and small size of the sensor, the fingerprint ridges are blurry and the impressions from the same finger usually have small overlapped area. The fingerprints are collected from heterogeneous volunteers including manual workers and elderly people, so the majority of them suffer the influence from blur, scar, wetness or dryness.

The experiments are conducted on three databases (DB1, DB3 of FVC2004 and our established database). All the matching performance in this section is evaluated according to the experimental protocols in [12]. To approve the efficacy of the combined features, we display the performance of the ridge-based method [5] and the minutiaebased method [1] for comparison in Table 2. The results confirm that the combined features can significantly improve the performance of fingerprint matching, especially for the poor-quality and partial fingerprints.

Table 2. Comparison of performance between our algorithm and other matching methods

\begin{tabular}{c|c|c|c|c|c}
\hline & & EER(\%) & FMR100(\%) & FMR1000(\%) & ZeroFMR(\%) \\
\hline \multirow{3}{*}{ DB1 } & Minutia-based & 5.74 & 9.35 & 15.54 & 18.43 \\
\cline { 2 - 6 } & Ridge-based & 4.57 & 6.71 & 16.10 & 31.18 \\
\cline { 2 - 6 } & Our algorithm & 3.49 & 6.21 & 19.04 & 24.61 \\
\hline \multirow{3}{*}{ DB3 } & Minutia-based & 2.78 & 4.47 & 8.40 & 11.86 \\
\cline { 2 - 6 } & Ridge-based & 2.11 & 3.04 & 8.75 & 16.86 \\
\cline { 2 - 6 } & Our algorithm & 1.31 & 1.61 & 4.29 & 9.15 \\
\hline $\begin{array}{c}\text { Our } \\
\text { established } \\
\text { database }\end{array}$ & Minutia-based & 6.55 & 10.89 & 17.54 & 39.18 \\
\cline { 2 - 6 } & Ridge-based & 5.01 & 5.51 & 8.28 & 30.69 \\
\cline { 2 - 6 } & Our algorithm & 3.33 & 4.47 & 19.39 & 33.54 \\
\hline
\end{tabular}

\section{Conclusion}

In this paper we represent the fingerprint with a novel local feature descriptor, which is comprehensive and robustly holds the uniqueness of the fingerprint. We align the fingerprints using the descriptors and calculate a set of transform vectors as candidates. In matching process, the orientation field is used to avoid misaligning and to measure the similarity score of global ridge pattern. We also evaluate the similarity between the local descriptor sets from the input and template fingerprints. The two methods are fused with the Sum rule to obtain the final matching score. The experimental results demonstrate that our algorithm integrates the advantages of the local and global features and improves the algorithm performance and reliability. 
Acknowledgments. This paper is supported by the Project of National Science Fund for Distinguished Young Scholars of China (No. 60225008), the Key Project of National Natural Science Foundation of China (No. 60332010 and 60575007), the Project for Young Scientists' Fund of National Natural Science Foundation of China (No.60303022), and the Project of Natural Science Foundation of Beijing (No.4052026).

\section{References}

1. Jain, A.K., Hong, L., Bolle, R.: On-Line Fingerprint Verification. IEEE Trans. Pattern Analysis and Machine Intelligence 19(4), 302-314 (1997)

2. Kovcs-Vajna, Z.M.: A Fingerprint Verification System Based on Triangular Matching and Dynamic Time Warping. IEEE Trans. Pattern Analysis and Machine Intelligence 22(11), 1266-1276 (2000)

3. Tian, J., He, Y.L., Chen, H., Yang, X.: A Fingerprint Identification Algorithm by Clustering Similarity. Science in China Ser. F Information Sciences 48(4), 437-451 (2005)

4. Feng, J.J., Ouyang, Z.Y., Cai, A.: Fingerprint Matching Using Ridges. Pattern Recognition 39, 2131-2140 (2006)

5. Gu, J.W., Zhou, J., Yang, C.Y.: Fingerprint Recognition by Combining Global Structure and Local Cues. IEEE Transaction on Image Processing 15(7), 1952-1964 (2006)

6. Tico, M., Kuosmanen, P.: Fingerprint Matching Using an Orientation-based Minutia Descriptor. IEEE Trans. on Pattern Analysis and Machine Intelligence 25(8), 1009-1014 (2003)

7. Chen, Y., Dass, S.C., Jain, A.K.: Fingerprint Quality Indices for Predicting Authentication Performance. In: Proc. of Audio- and Video-based Biometric Person Authentication (AVBPA), pp. 160-170. Rye Brook, NewYork (2005)

8. Luo, X.P., Tian, J.: Image Enhancement and Minutia Matching Algorithm in Automated Fingerprint Identification System. Journal of software 13 (2002)

9. Kittler, J., Hatef, M., Duin, P.W., Matas, J.: On combining classifiers. IEEE Trans. Pattern Analysis and Machine Intelligence 20(3), 226-239 (1998)

10. Ho, T.K., Hull, J.J., Srihari, S.N.: Decision combination in multiple classifier systems. IEEE Trans. Pattern Analysis and Machine Intelligence 16(1), 66-75 (1994)

11. http://bias.csr.unibo.it/fvc2004/

12. Maio, D., Maltoni, D., Cappelli, R., Wayman, J.L., Jain, A.K.: FVC2004: Third Fingerprint Verification Competition. In: Zhang, D., Jain, A.K. (eds.) ICBA 2004. LNCS, vol. 3072, pp. 1-7. Springer, Heidelberg (2004) 